\title{
Finalités, valeurs et identités pour fonder une éducation écocitoyenne
}

Marie-Louise Martinez et Frédéric Poydenot

\section{(2) OpenEdition \\ Journals}

Édition électronique

URL : http://journals.openedition.org/ere/2128

DOI : $10.4000 /$ ere. 2128

ISSN : 2561-2271

Éditeur

Centr'ERE

Référence électronique

Marie-Louise Martinez et Frédéric Poydenot, «Finalités, valeurs et identités pour fonder une éducation écocitoyenne», Éducation relative à l'environnement [En ligne], Volume 8 | 2009, mis en ligne le 20 décembre 2009, consulté le 21 février 2020. URL : http://journals.openedition.org/ere/2128; DOI 10.4000/ere.2128

Ce document a été généré automatiquement le 21 février 2020. 


\title{
Finalités, valeurs et identités pour fonder une éducation écocitoyenne
}

\author{
Marie-Louise Martinez et Frédéric Poydenot
}

1 L'éducation à l'environnement (EE), au-delà des disciplines académiques concernées comme la géographie, les sciences de la vie, ou l'économie, parce qu'elle touche aux valeurs, au sens et aux finalités, mobilise de plus en plus les sciences de l'éducation dans leurs approches philosophiques ou anthropologiques.

2 L'écocitoyenneté et l'écoresponsabilité comme visées politiques et éthiques de l'éducation à l'environnement ont en commun d'être à la fois des finalités, des valeurs et même des caractéristiques perceptibles d'une identité personnelle et sociale à construire. Elles impliquent de questionner à nouveaux frais, le rapport à soi, à l'autre, à la cité, au monde, elles demandent de revisiter le sujet de l'éducation, de repenser les pratiques éducatives autant que les démarches de la recherche en éducation. Elles demandent un modèle anthropologique de l'éducation.

3 Nous proposons ici l'esquisse d'un modèle théorique d'anthropologie relationnelle de la personne en philosophie de l'éducation dont la construction se poursuit depuis plus de 10 ans (Martinez 1997, 2003 et 2009) et qui s'appuie ici sur une étude plus ponctuelle, en réponse à un appel d'offre de l'Agence de l'Environnement et de la Maitrise de l'Énergie (ADEME) et de la Région Provence-Alpes-Côte d'Azur (2006-2008). Cette recherche a consisté dans le suivi des effets de quelques activités éducatives du CPIE (Centre permanent d'initiatives pour l'environnement) des îles de Lérins et Pays d'Azur (à Cannes).

4 Nous ébauchons le cadre d'une anthropologie philosophique de l'éducation qui articule en convergence diverses branches: didactique ${ }^{1}$ (contenus à transmettre), pédagogique (méthodes), axiologique (visées et finalités), éthique (valeurs), praxéologique (pratiques). En précisant les notions, nous situons les diverses dimensions du modèle :

- éducation à l'écocitoyenneté comme finalité axiologique ;

- éducation de la personne comme valeur éthique et modèle du sujet écoresponsable qui intègre le tiers précédemment exclu ; 
- éducation par des pratiques éducatives où se co-construisent les identités à travers les processus d'identification (appartenance et différence).

5 Enfin, nous évoquons le cadre théorique et la démarche méthodologique pour apprécier les conditions et les effets de ce processus d'identification: l'approche clinique et collaborative mise en œuvre pour observer et évaluer la construction des identités au sein des échanges langagiers de la pratique éducative. Nous avons suivi des pratiques d'animation auprès d'enfants du primaire dans le cadre de semaines découvertes et auprès d'adolescents en collège, dans le cadre de la conduite de projets pédagogiques, pour évaluer la prise de conscience "environnementale» et la construction identitaire.

6 L'écoresponsabilité et l'écocitoyenneté, en effet, ne peuvent simplement se concevoir comme des "comportements", externes au sujet et bien souvent conditionnés, elles demandent à être comprises comme des "conduites", des "identités " à la fois singulières et sociales, construites au cours de la pratique et de l'action partagée. Aujourd'hui, c'est dans l'interaction et la relation qu'il faut penser la construction de savoirs, de conduites et d'identité, le sujet n'est pas conformé de l'extérieur, le développement n'est pas pour autant secrété et mu depuis le for intérieur subjectiviste. Le développement des connaissances, des identités s'opère dans l'espace interlocutif et dialogique de la rencontre et des pratiques éducatives, comme des co-constructions dans l'entre deux (ou plus). En tant que telles, elles sont repérables et observables selon leurs caractéristiques dans les pratiques langagières. Quelles marques langagières, indicateurs d'une construction identitaire écoresponsable et écocitoyenne, peut-on retrouver dans les propos d'enfants et d'adolescents engagés dans les pratiques éducatives conduites par les animateurs du CPIE?

\section{Une anthropologie philosophique pour l'éducation écocitoyenne}

7 L'axiologie, l'éthique et la praxéologie, comme branches du questionnement philosophique, concernent les valeurs, les principes et les fins, comme autant de concepts définis par ce domaine disciplinaire.

\section{L'axiologie comme interrogation sur les finalités désirables}

8 L'axiologie concerne le questionnement sur les finalités et la fin qui doivent prévaloir. Quelquefois explicites, la plupart du temps implicites, les finalités tiennent lieu de fondement à l'action éducative. On les trouve dans les discours, les projets et les réformes éducatives. La finalité implique la visée et le sens attribués à l'action. Elles sont aujourd'hui de plus en plus escamotées au profit de la description plus instrumentale de moyens servant à atteindre des objectifs opérationnels. C'est aux finalités de l'école et aussi à l'écart entre les finalités déclarées et les priorités effectives qu'on mesure la qualité éducative d'un système éducatif. Elles permettent de repérer quelles caractéristiques on attend de l'élève éduqué, quel Homme on veut former. Vise-t-on le citoyen éclairé, émancipé, conçu par les précurseurs (Rousseau, Kant) ou les fondateurs de l'école de la République (Ferry, Buisson, Durkheim, etc.), ou plutôt un consommateur adapté à la société, un travailleur aux compétences utiles, socialement efficaces et transférables, répondant aux besoins flexibles du marché, comme le suggère 
l'Organisation de coopération et de développement économiques (OCDE) ? La question des finalités dévoile le modèle de société en jeu, seules les finalités sont capables de mobiliser le sens dans l'action et de dynamiser les acteurs ${ }^{2}$. Veut-on émanciper des individus au sens fort du terme comme sujets à la fois plus autonomes et solidaires ou au contraire souhaite-t-on continuer à fabriquer d'un côté des membres plus compétitifs et de l'autre, des exclus, désaffiliés (Castel et Haroche, 2001) du lien social?

erojet de société sous-tendu par l'éducation à l'environnement et pour un développement durable demande de repenser le modèle de citoyen et d'être humain véhiculé par les pratiques éducatives qu'elles soient institutionnelles ou associatives. Seront-ils adaptables et employables selon un référentiel globalisé de compétences transférables, capables de servir «durablement » les objectifs utilitaristes de la société ultra libérale, des producteurs consommateurs précarisés, dociles à reproduire les gestes et les réponses préconçus martelés par la propagande médiatico-politique à des problèmes hérités? Désire-t-on plutôt développer un citoyen critique instruit par des savoirs plus universalisables, capable de poser de nouvelles questions et de les résoudre dans la concertation? Vise-t-on enfin l'émergence de l'élève comme écocitoyen susceptible d'inventer de nouvelles problématiques, comme personne écoresponsable, dans une société démocratique où les politiques publiques s'appuieraient sur l'initiative des acteurs?

\section{Hiérarchie des finalités et de la fin}

11 La question de la finalité est celle de l'horizon désirable en vue de quoi tendent les actes. La fin plus ultime encore que les finalités, absolu visé par l'action, signale le bien désirable selon les attributions d'une institution et lui donne son sens (aux deux sens du terme). La question des finalités de l'action, dans le cadre de l'institution juste, est une question de philosophie politique. La fin est l'équivalent philosophique des notions de but (géographie) ou de cible (balistique), de visée (stratégie), d'horizon (géométrie), etc. Elle n'apparaît que dans quelques philosophies ou visions du monde finalistes ou téléologiques (telos gr. fin). Les pensées téléologiques sont toutes liées à une philosophie de l'histoire comme temps orienté vers un devenir et porteur de sens, elles sont aujourd'hui critiquées par les courants post-modernes qui récusent la notion de sens et d'histoire. Mais peut-on évacuer les questions de sens et d'histoire en éducation?

En outre, l'écologie donne une portée brûlante à la question des finalités, parce qu'elle demande de repenser le lien entre la culture et la nature. Le dualisme $e^{3}$ de l'humanisme classique qui conçoit le corps et l'esprit, la nature et la culture, l'être humain et l'animal, etc. comme des réalités mutuellement exclusives est critiquable dans ses effets. À la lumière de la crise écologique, on l'accuse (Jonas) d'avoir encouragé un anthropocentrisme destructeur. Mais le monisme comme vision de l'unité, tantôt spiritualiste, tantôt matérialiste, fondé sur une conception immanente et fusionnelle, pourrait bien englober l'Homme dans un grand tout cosmique sans perspective «d'arrachement à son milieu » ni de liberté. Luc Ferry (1992), entre autres, a critiqué certains courants actuels de l'écologie qui puiseraient, selon lui, leur inspiration dans une conception qui, en l'absence d'extériorité ontologique, impliquerait une forme de régression fusionnelle naturiste et anti humaniste.

13 Aujourd'hui, la crise écologique et l'enjeu de l'éducation à l'environnement viennent redonner à ces questions philosophiques et ontologiques l'importance qu'elles ont toujours eue, mais qu'on avait trop perdue de vue. Les controverses actuelles entre les différents courants de l'écologie et leurs détracteurs méritent l'attention. Elles 
manifestent les incidences majeures politico-anthropologiques des représentations métaphysiques que chacun véhicule et qu'il importe de repérer (l'impensé et le refoulé, chassés par la porte, reviennent toujours par la fenêtre). Le dualisme a encouragé un rationalisme technologique qui aurait "désenchanté", selon le mot de Max Weber (1905), et instrumentalisé le monde au mépris des équilibres vitaux, mais le monisme prôné aurait de quoi inquiéter par les risques fusionnels et les tentations totalitaires qu'ils impliquent.

14 Émile Durkheim, optait pour le dualisme, avec des accents pascaliens :

[...] l'Homme est à la fois « ange et bête » sans être exclusivement ni l'un ni l'autre.

[...] C'est ce désaccord, cette perpétuelle division contre nous-mêmes qui fait, à la fois, notre grandeur et notre misère. [...] Ainsi l'antithèse traditionnelle du corps et de l'âme n'est pas une vaine conception mythologique, sans fondement dans la réalité. Il est bien vrai que nous sommes doubles que nous réalisons une antinomie. (Durkheim, 1975a, p. 320-321)

Peut-être doit-on reconnaître l'humain dans sa réalité double et paradoxale sur le plan anthropologique, être de tension entre l'individu et le social, entre la culture et la nature. Il me semble que la juste vision du monde est relationnelle, ni moniste ni dualiste, mais dialogale. L'antinomie radicale pourrait se dépasser par l'émergence de la personne qui assume les règles, les lois et les limites exigées par l'appartenance à la cité, et à l'écocité, sujet réflexif écoresponsable, qui respecte en elle et en l'autre (l'autre humain et environnement) l'appartenance à la nature et à la culture.

Cette mise en relation sans confusion de deux ordres distincts, mais reliés permettrait de renvoyer dos à dos les idéologies créationnistes et nihilistes qui reviennent en force aujourd'hui. Les uns maximisent la finalité qu'ils confondent avec le finalisme biologique et sont prêts à contester la théorie scientifique de l'évolution des espèces. Les autres, au contraire, ont évacué tout questionnement des finalités et du sens au nom de l'utilitarisme. Or, la finalité ou la fin, trop négligées au profit des objectifs, impliquent l'intentionnalité et le sens qui sont attribués dans l'action éducative, anthropologique, ou politique par l'acteur. Il importe aujourd'hui que le citoyen et le sujet puissent revisiter les finalités de manière critique et réflexive.

Les seules finalités valables pour l'écologie restent l'« intérêt général » et le «bien commun", encore s'agirait-il de les penser de manière humaniste, mais sans anthropocentrisme contre-productif. En outre, pour éviter les dérives d'un contrôle social et politique abusif, il importe, à côté de la finalité politique de l'écocitoyenneté, de faire place à la fin de l'émergence de la personne écoresponsable. L'impératif kantien, pour éviter l'instrumentalisation de la personne au service d'une quelconque finalité, demande «que tu traites l'humanité aussi bien dans ta personne que dans la personne de tout autre toujours en même temps comme une fin, et jamais simplement comme un moyen " (Kant, 1785). La fin du respect de la personne semble, en effet, devoir être placée au-delà de toute finalité, fut-elle la plus "vertueuse ». Cette hiérarchie entre finalités et fin me semble indispensable dans un domaine de politiques publiques où bien vite (on ne l'a que trop vu) on est amené à céder aux tentations totalitaires : on peut alors justifier le sacrifice de certaines personnes à ce qu'on estime être l'intérêt général.

\section{La personne comme seule fin pour l'éducation}

19 La personne n'est pas l'individu indépendant, égocentrique, isolé ou esseulé du corps social. Elle se construit dans, par et pour la relation comme sujet réflexif, elle a 
intériorisé les limites du respect de la loi et la conscience de sa dépendance et de sa responsabilité à l'égard de l'autre et de l'environnement. La personne émerge à travers les interactions langagières par la médiation des pratiques, dans un processus d'(inter)identification et de différenciation qui le rapproche et le distingue de l'autre humain (Jacques, 1982, 1985 ; Ricœur 1990), et de l'environnement.

Pour viser la fin de l'émergence de l'élève comme personne, l'école considère habituellement trois finalités essentielles :

a. la transmission / l'appropriation critique de savoirs pertinents (ici, ceux du corpus systémique du développement durable) comme ensemble de médiations académiques et de pratiques éducatives ;

b. l'éducation (éco)citoyenne - La citoyenneté, participation politique à la cité, ciment du lien social en démocratie, renvoie à la transcendance laïque d'un intérêt général et d'une chose publique à protéger ensemble. L'éco-citoyenneté (du gr. oikos, habitat) est un néologisme créatif et frappant qui a son utilité. La notion est redondante si l'on considère que toute véritable citoyenneté implique l'indispensable partage d'un bien commun élargi à l'altérité intergénérationnelle, intercommunautaire, interspécifique. La notion est aporétique sinon utopique si elle implique un lien contractuel ou politique avec le non humain (Serres, 1990).

c. Les deux précédentes concourent à la troisième : l'émergence d'une identité de sujet réflexif comme personne, « souci de soi, de l'autre, et de l'institution juste » (Ricœur 1990).

Mais si la personne est fin pour l'axiologie, elle est encore valeur pour l'éthique.

\section{L'éthique comme savoir sur les valeurs}

22 Ethos (gr.) et mores (lat.) désignent tous deux les mœurs, mais l'éthique est davantage en surplomb des morales particulières qui ont été relativisées. La référence à l'éthique devient un enjeu majeur, dans les sociétés multicommunautaires où règne le pluralisme des valeurs et des normes, ou dans les sociétés en crise des repères, quand il est difficile de fonder en raison une valeur comme universelle. L'éthique environnementale est discutée dans ses fondements depuis longtemps par la philosophie ou l'anthropologie politique (Heidegger, 1953 ; Arendt, 1957 ; Jonas, 1985 ; etc.), aujourd'hui, elle doit l'être au sein de l'éducation. La personne pourrait bien être une valeur, vraiment relationnelle et universelle, au-dessus de tout soupçon, pour fonder l'éducation.

\section{Pour une autonomie d'autolimitation}

Hans Jonas (1985) voit dans les initiateurs de la modernité techno-scientifique, comme Descartes ou Hobbes ${ }^{4}$, les chantres d'un individualisme jouisseur aux effets suicidaires, l'humanité dégrisée devra, selon lui, affronter la dette contractée par « la fête frivole et joyeuse de quelques siècles industriels payée par des millénaires d'un monde transformé » (Jonas, 1985, p. 253). Hans Jonas (1985) prône une éthique et une politique d'austérité et de décroissance.

Un siècle plus tôt, Émile Durkheim, fondateur des sciences humaines en France, semble moins pessimiste. Il est vrai qu'il est mort en 1917, avant la grande crise et les totalitarismes. Sa supériorité et la grande actualité de sa pensée et de son message, aujourd'hui sont mises en relief par des relectures décapantes. Elles consistent surtout dans l'analyse des processus relationnels et anthropologiques de la crise et dans les pistes qu'il présente par sa théorie de l'éducation pour les surmonter. 

(Durkheim, 1998), en appelait déjà à la modération et à l'autolimitation (Durkheim, 1992) pour viser l'autonomie véritable dans l'interdépendance reconnue. On n'accède selon lui à la liberté qu'en acceptant les contraintes ${ }^{5}$, par l'intériorisation critique des normes et des limites, l'individu devient sujet (étym. subjectum: soumis), par la soumission librement consentie à l'impératif moral et il devient pleinement personne, en considérant l'autre justement comme une personne, c'est-à-dire « comme un fin et jamais comme un moyen ». Émile Durkheim $(1992,1998,1999)$ définit la notion d'anomie (a-nomos : absence de règle et a-nomein: absence de limite) pour décrire les abus et dérives de l'individualisme ${ }^{6}$. Tant qu'il ne prend pas conscience de l'insatiabilité de son désir, l'individu succombe à ce que l'auteur nomme le «mal de l'infini», perte des limites et des régulations, économiques (Durkheim, 1998), politiques, sociales ou psychiques (Durkheim, 1999) et éducatives (Durkheim, 1992). Pour lui, l'anomie provient d'une indifférenciation identitaire, consécutive à la basse des régulations et au déclin des institutions. La solution ne peut venir que de la structuration de corps intermédiaires (associations scientifiques et citoyennes), pour différencier les sujets et permettre un lien social complémentaire et solidaire et de l'éducation de la personne.

fectivement, on s'aperçoit aujourd'hui de la justesse des analyses d'Émile Durkheim, l'individualisme dérégulé peut dissoudre le lien solidaire à la communauté et à l'autre, entraîner désaffiliation et précarisation, ce que Castel et Haroche (2001), appellent «l'individu par défaut». L'antidote serait la personne ${ }^{7}$ : autonome et solidaire, consciente de l'interdépendance à l'autre et à l'environnement, responsable. Pour Émile Durkheim, l'individu comme personne demeure la seule fin possible ${ }^{8}$, le seul sacré 9 partageable dans une société plurielle et laïque, susceptible d'être placé au fondement de l'école et de toutes les institutions.

Mais l'impératif catégorique revisité par Hans Jonas (1985) « Agis de telle façon que ton action soit compatible avec la permanence de la vie sur terre " pose la question des autres formes de vie, la personne comme sujet en relation peut-elle fonder un humanisme non anthropocentrique?

La philosophie interlocutive (Jacques, 1985 ; Ricœur, 1990) inscrit la construction de la personne, dans la pratique langagière: processus d'intersubjectivation à travers la capacité tri-instancielle (je, tu, il) du pronom personnel. Pour advenir pleinement comme personne, on doit pouvoir s'identifier au tiers proche ou lointain, lui faire une place avec compassion, mais sans confusion. Le sacrifice du tiers (Girard, 1982, 2004) soude le consensus des communautés culturelles archaïques et signe encore aujourd'hui les identitarismes sectaires ou la violence totalitaire. La personne, sujet responsable de l'autre comme tiers (Lévinas, 1991) refuse le pacte symbolique (Martinez, 2003) qui repose sur son éviction.

Le linguiste Émile Benveniste (1966) a montré comment le «il» comme «nonpersonne $»^{10}$ fonde la langue et sans doute la culture. L'autre (étranger, pauvre, groupe humain différent, nature, animal, corps, etc.) n'a-t-il pas trop souvent joué le rôle de tiers exclu sur l'éviction duquel repose la culture comme système sacrificiel (Serres, 
1990 ; Girard, 2004) ? Dès lors, à la lumière de la crise écologique, l'enjeu de l'éducation de la personne comme sujet réflexif qui accueille l'autre devient vital. comme fin et valeur. La réflexion praxéologique demande des pratiques éducatives, en cohérence avec les fins visées et les valeurs défendues. S'il s'avère indispensable de transmettre des valeurs, on ne saurait les « inculquer » (étym. : faire rentrer à coups de talons). Il ne s'agit pas seulement de prescrire un comportement adapté, des bons gestes, même s'ils ont leur intérêt. Legardez et Simonneaux (2007) et Legardez (2008) proposent d'éduquer aux choix plutôt que d'enseigner des choix, en travaillant sur le débat autour de «questions socialement vives ». Il faut permettre des rencontres, des situations, des praxis ${ }^{11}$ où se développent ensemble les savoirs, les ressources vitales et identitaires, subjectives et sociales, avec la capacité à faire des choix.

cœur de ces pratiques, se développent les compétences identitaires et psychosociales discursives, relationnelles, en fonction de l'espace social de référence et du milieu. Lucie Sauvé l'a bien repéré :

[...] l'éducation relative à l'environnement a certes un rôle important à jouer pour le développement identitaire. On sait que le moi, le je, singulier ou collectif, se construit dans le rapport à l'autre humain. Mais il se construit aussi dans le rapport au milieu de vie, le rapport à Oökos, cette maison de vie, partagé. Notre identité psycho-sociale est tissée à même notre «identité écologique » (Carvallo, 2004), celle qui se construit dans l'interaction avec le milieu (la maison, l'habitat urbain, le village, la biorégion, etc.) et qui nous relie à la terre, à l'eau, aux paysages, aux autres vivants, celle qui nous situe dans la trame de la vie partagée. (Sauvé, 2006-2007, p. 22)

Les activités dans les îles, les projets pédagogiques, animés par le CPIE, à travers des pratiques issues de l'éducation populaire, offrent un vaste laboratoire de coconstruction des identités de personnes écoresponsables et écocitoyennes. La pédagogie du projet transforme l'élève en sujet qui participe par son intentionnalité, son désir, à une activité avec d'autres où il trouve du sens. La pratique coopérative et ludique avec les autres enfants, dans un cadre structurant assuré par les adultes animateurs, permet la découverte de l'environnement, développe la solidarité et la responsabilité. 


\section{Une anthropologie clinique pour la co-construction des identités}

39 Ces activités permettent la rencontre sensorielle, expérientielle, émotionnelle avec d'autres (enfants, préadolescents, adultes, paysages et milieu vivant) à l'occasion d'un séjour exceptionnel ou dans la fréquence hebdomadaire d'un projet à l'année. Les paramètres de la rencontre (densité des corps et du silence, variation des temporalités, présence animale, végétale, odeurs, gestes) participent totalement à l'épaisseur sémiotique des pratiques langagières. L'identité écocitoyenne et écoresponsable de la personne se «fabrique » là au sein des interactions, dans un tissu tressé d'imaginaire, de perceptions, d'émotions, de passions, de savoirs, de prises de conscience.

Il s'agit, par le jeu toujours imprévisible de ressemblances et dissemblance aux modèles identificatoires, de provoquer l'adoption d'aptitudes, de capacités, de compétences, de croyances, subjectives psychoaffectives et psychosociales particulières, d'inciter un rapport à soi, à l'autre, à la cité, à l'environnement, marqué par le sentiment de vulnérabilité, mais aussi de confiance qui engage un désir d'agir avec et pour d'autres.

41 Les notions évoquées (sujet, personne (éco)responsable, (éco)citoyen) ne sont pas simplement des notions philosophiques abstraites et théoriques, mais des visées axiologiques, valeurs éthiques et praxéologiques pour penser l'action. Nous faisons l'hypothèse qu'elles sont une série de processus et de dynamiques, mais qui se remarquent dans l'instant comme des qualités et caractéristiques qui peuvent se retrouver dans l'identité singulière et sociale des personnes empiriques. Elles peuvent donc en partie s'observer, se décrire, se constater comme des "faits sociaux", c'est pourquoi notre cadre théorique articule une anthropologie philosophique (examen théorique des finalités et du sujet de l'éducation) et une anthropologie scientifique (étude empirique et pratique des identités et de leur construction dans les interactions langagières dans les situations concrètes).

\section{L'approche anthropologique de la question de l'identité}

Les identités ne sont pas des substances qui préexistent (âme, origine, caractère, nature, etc.) qu'il s'agirait de retrouver et d'exprimer. Elles sont plutôt des processus en perpétuelle construction, à partir de certaines données initiales (biologiques, génétiques, sociales, affectives, etc.), mais toujours réinterprétées et remaniées par une dynamique dans la relation et les interactions. Les identités se construisent dans les interactions avec le milieu et les autres, dans une mimésis très puissante chez l'Homme, sans doute encore plus forte que pour les autres animaux, compte tenu de la néoténie de l'humain due à sa fragilité à la naissance et à sa longue enfance. La capacité imitative de l'Homme est sans doute bien supérieure à celle des autres animaux, car elle ne concerne pas seulement les attitudes et les comportements externes, elle touche plus intimement l'imitation de l'autre proche, dans ses conduites, ses manières d'être, de désirer et d'avoir. La mimesis acquisitive chez l'Homme est appropriative, désirante, elle produit la grande intelligence humaine dans les apprentissages, mais elle provoque aussi un processus d'identification à l'autre toujours menacé par le fusionnel ou le rivalitaire : l'identité est toujours liée à l'autre auquel on s'identifie en miroir. Cette mimesis désirante inouïe est la force de l'humain et sans doute la clé du succès de l'espèce humaine dans son évolution. C'est aussi sa grande vulnérabilité, l'identification à l'autre et à son désir (amour, avoir, être) bute sur la rivalité réciproque éminemment 
destructive qu'il faut réguler. Dans les sociétés traditionnelles, un ensemble de rites, d'interdits et d'institutions fortes dans un système religieux (religere: créer du lien social) permet d'assigner et différencier clairement les identités. Les sociétés modernes critiquent le système sacrificiel et veulent l'égalité et la liberté de l'individu. Plus justes et plus démocratiques, nos sociétés sont aussi plus vulnérables et violentes parce qu'elles dérégulent leurs institutions, elles déstabilisent et désenchantent le lien social, elles rendent la construction identitaire plus incertaine. Nos analyses s'inspirent de l'anthropologie mimétique de René Girard $(1982,2004)$, qui coïncident largement avec celles de Émile Durkheim $(1998,1999)$ sur l'anomie et l'indifférenciation. Les solutions envisagées par Émile Durkheim, tournent essentiellement autour d'une éducation qui permette la construction d'identités différenciées, responsables et solidaires. C'est pourquoi le défi éducatif actuel de la construction d'identités de personnes et de citoyens écoresponsables nous semble en mesure de répondre à la crise anthropologique et à la crise écologique.

On n'a jamais autant parlé d'identité que depuis que chacun est confronté à l'autre qui se rapproche dans son statut social et sa condition, sur le plan mondial. L'exigence identitaire est d'autant plus forte que chacun dans une société complexe a une identité plurielle, incertaine et bricolée. L'identité se construit dans un rapport avec autrui, dans la relation qui conjugue avec subtilité identification et distinction, appartenance et singularité, différence et ressemblance, demande de reconnaissance et besoin de distance ou de dissidence. Elle est constituée par un ensemble de marques attribuées par soi et par autrui, dans la reconnaissance ou dans le stigmate comme marquage infamant du regard sur l'autre (Goffman, 1963).

Les caractéristiques identitaires sont invisibles et visibles : biologiques (ADN, âge, sexe, couleur de peau, traits physiques), familiales, administratives ou civiques (statut familial, nom, prénom, contexte et géographique, national et familial). À partir de traits plus ou moins fixes, physiologiques, historiques, sociaux et culturels, groupe d'appartenance et groupe de référence, langue maternelle, elles sont réinterprétées (roman de l'origine, récit de vie propre, histoire familiale ou ethnique ou nationale). Leur construction est dynamisée par des forces psychoaffectives, psychosociales et cognitives (désir, imaginaire, systèmes de valeurs, de références symboliques, religieuses, philosophiques et scientifiques), etc. La plupart des identités sont à la fois collectives, liées à des appartenances (familiales, politiques, nationales, professionnelles, etc.) et singulières, sentiment d'être unique, lié à des perceptions intimes dans leur combinaison. L'identité harmonieuse répondrait à la satisfaction toujours instable de ce double besoin d'appartenance et d'unicité.

\section{La lecture discursive des marques langagières}

La "fabrication" de ces identités se négocie au cœur des interactions langagières, au sein de la pratique. On peut dès lors en repérer et en étudier les traces et les indicateurs dans le discours interlocutif. Pour saisir la construction identitaire à l'œuvre et en permettre l'objectivation et l'étude, il faut d'abord prélever, recueillir, transcrire les échanges langagiers par enregistrement audio ou audiovisuel. La démarche d'analyse interlocutive (de locare : parler), bien développée depuis des années par la linguistique, la philosophie du langage et la sociologie interactionniste, permet de scruter les indicateurs identitaires (lexique, syntaxe, l'énonciation, actes de langage, tours de paroles, répétitions, rites d'interaction, silences, balbutiements, chevauchements, etc.) dans l'échange langagier. 

acquis et construits, mais aussi la capacité morpho-syntaxique d'utiliser divers discours, et enfin les indicateurs pragmatiques, directement subjectifs et relationnels de l'énonciation. qui composent un parler spécifique, lexique sur l'environnement qui leur conférait l'appartenance à une communauté discursive, savoirs appropriés de façon non livresque, mais désignés par des concepts précis. Lorsqu'ils parlent de la "posidonie " comme d'une plante et qu'ils convoquent à propos les concepts de classification des espèces, les enfants se sentent valorisés et distingués dans leur singularité. Ils ont conscience de développer une "identité discursive » avec l'usage de concepts et d'un lexique spécifique et partagé dans le groupe, mais aussi par la communauté scientifique qui est universelle.

L'entrée morpho-syntaxique, nous a permis de repérer la capacité des enfants à entrer dans un discours descriptif, narratif, explicatif, voire argumentatif, avec plus ou moins de cohérence et d'à-propos. Ces capacités discursives peuvent être mises en relation avec les compétences psychoaffectives et psychosociales identitaires.

51 Enfin, l'entrée interlocutive et énonciative, centrée sur le repérage des pronoms personnels, des mots exprimant l'affectivité ou l'attitude subjective et relationnelle du sujet, sa manière de concevoir ou d'interpeller l'autre, a été très fructueuse pour saisir l'identification singulière et relationnelle. démarche clinique inventée par les médecins qui constataient que l'élaboration des savoirs se faisait au chevet du malade, autant que dans la recherche expérimentale en laboratoire, s'est répandue dans les sciences humaines qui veulent saisir la singularité des sujets et des situations, en enquêtant auprès des acteurs sur ce qu'ils montrent et disent de leur vécu. La démarche est aussi collaborative, c'est-à-dire que les acteurs, ici, les animateurs de l'environnement, participaient à la recherche et que de son côté le chercheur, impliqué, analysait lui-même son rapport à l'objet étudié. années, donnent à voir l'ampleur du travail éducatif réalisé. Effectués par les animateurs sous forme d'entretien avec des petits groupes, ils se présentaient comme un sondage auprès des enfants sur les apprentissages occasionnés. Les enfants ont acquis des savoirs nouveaux sur le plan académique, et méthodologique, mais surtout sur le plan communicationnel, social et identitaire. Ils s'incluent dans des groupes d'appartenance avec les relations fortes qui les constituent, marquées par les milieux fréquentés (fort de l'île, plages, mais aussi famille, classe, école) impliquant l'affect, l'intellect et l'appropriation des normes.

On perçoit chez eux la joie de se situer plus clairement, le désir de partager l'affiliation ou au contraire de se démarquer. $M$ est content de dire la démarche, les valeurs qui différencient son groupe et sa classe des autres: Parce que nous ce qui nous a différencié d'autres classes, c'est parce que NOUS on avait un projet. La classe environnement c'est pas seulement quelque chose où on apprend LA MER ET TOUT, on doit apprendre travailler en groupe à être solidaires). 

s'entrechoquer. Ma souligne par l'emphase les thématiques et les valeurs découvertes : Moi c'est pour lutter contre la POLLUTION, les animaux en voie de DISPARITION, la mer, pour connaître mieux les espèces et puis aussi parce que ça me plaît. Elle montre aussi qu'elle y adhère avec plaisir, de plein gré. L'émotion est perceptible, elle accompagne un processus d'initiation à une identité nouvelle et partagée, par l'accès à des concepts valorisés et religieusement partagés. Les notions acquises sont saluées et citées avec dévotion, mais ici, la restitution fidèle chez $M a$ est sans doute la phase préalable indispensable à une réappropriation singulière. On redoute souvent que ces enfants ne subissent, à travers l'éducation à l'environnement, ou plus encore au développement durable, une sorte de catéchisme modélisateur, de conformation mimétique. Nous avions effectivement quelquefois l'impression que les enfants citaient un discours appris: «je faisais tout ça pour lutter contre les pollutions, les animaux en voie de disparition, la mer pour mieux connaître les espèces » disait, sans en être dupe, une jeune fille, mais, rajoutait-elle « et aussi parce que ça me plaît ».

Il est inévitable qu'un savoir transmis par des adultes avec des convictions devienne vite modélisateur. Le mimétisme en éducation est constitutif de la transmission, s'il ne devient pas endoctrinement confusionnel et que des dispositifs de réflexion, de construction des savoirs, d'instruction, de discussion, sont mis en place dans le temps long de la découverte, de l'expérience et de la discussion. Les enfants adhérent aux valeurs que le CPIE partageait avec eux, mais ils se positionnent comme des sujets parce que la distance critique et appropriative est favorisée par les situations de réflexion, par la discussion de thèses scientifiques. Ils ne se gênent d'ailleurs pas, pour critiquer les pratiques des animateurs et pour discuter âprement les thèses proposées.

On constate chez eux la prise de conscience des autres formes de vie, d'une identité commune avec les vivants des autres espèces vers l'empathie d'un destin partagé non dénuée souvent d'une certaine confusion anthropocentrique : Un arbre ça vit, ça évolue, ça produit ça puis ça fait des générations futures quoi, c'est comme un animal c'est juste en fait pour beaucoup de personnes les animaux vu que c'est plus mignon on va leurs apporter plus d'importance tandis que les arbres c'est pareil quoi ça vit c'est comme nous quoi.

Mais cette identification de soi à l'autre et de l'autre à soi est occasion d'éprouver le sentiment vif d'un engagement et d'appel à une responsabilité : Franchement moi je ne me vois pas me dire voilà quand je serai morte je n'aurai pas sauvé les autres là ce sera plus qu'un bout de roche avec plus personne dessus encore les plantes encore pourront revivre, mais nous il y aura plus d'espèces, mais si ça continue comme ça on va rester comme les DINOSAURES il y a aura une autre espèce de vie ce serait bête de tout gâcher.

On entrevoit ici une découverte personnelle et sociale, où l'autre participe à la construction d'une identité nouvelle élargie au tiers. On perçoit l'éveil à l'écoresponsabilité à l'égard des " autres sauvés », du destin de l'espèce en lien avec les règnes minéral, végétal, animal, etc. Ma se met en scène dramatiquement, elle-même comme l'espèce, qu'elle entend préserver du naufrage des dinosaures dans le temps d'un futur apocalyptique. Ces propos avec un vocabulaire et un univers symbolique spécifique véhiculent une conception du monde, définissent et construisent une véritable communauté discursive en référence à un champ spécialisé d'activités.

61 Il aurait été souhaitable de pouvoir observer dans le temps comment ces ressources nouvelles, sensorielles, imaginaires, symboliques, s'inscriront en profondeur dans l'identité personnelle. Cette recherche, par sa dimension descriptive, a permis de 
clarifier des hypothèses, de mieux les formuler même si ses limites majeures concernant la dimension longitudinale ne nous a pas encore permis de mesurer précisément l'évolution des enfants. Par la place accordée à la notion d'identité qui est un objet extrêmement heuristique, notre travail clinique a pu saisir et apprécier des moments identitaires, des investissements très forts, nous avons un recueil d'indicateurs de données identitaires qui vont donner des pistes sur les effets produits et à produire pour améliorer les pratiques éducatives. Nous entrevoyons mieux aussi comment améliorer les dispositifs d'une recherche empirique et clinique.

En conclusion, on peut dire que dans le contexte de crise et de menaces écologiques pesant sur la planète et ses ressources vitales, il importe de déconstruire l'individualisme anthropocentriste et de reconstruire un humanisme écoresponsable. Jonas (1985) nous enjoint à une "heuristique de la peur" susceptible de protéger l'avenir de l'humain, Jean Pierre Dupuy (2002) nous invite à un «catastrophisme éclairé » et Michel Serres (1990) à un « nouveau contrat naturel ».

Mais ces perspectives ne prendront corps qu'à partir de pratiques éducatives inventives qui permettront aux enfants des transformations identitaires profondes. Un tel chantier éducatif est à l'œuvre, entre autres dans les pratiques éducatives associatives, mais il attend la reconnaissance et l'étayage théorique.

Nous espérons avoir contribué, modestement, par la réflexion théorique et pratique à la refondation du sujet de l'éducation comme personne et à l'élaboration d'un modèle d'intelligibilité de la construction des identités écocitoyennes et écoresponsables dans les échanges langagiers.

\section{BIBLIOGRAPHIE}

Arendt, H. (1957, traduction française 1972). La crise de la culture. Paris : Gallimard. Benveniste, E. (1966). Problèmes de linguistique générale. Paris : Gallimard.

Castel, R. et Haroche, C. (2001). Propriété privée, propriété sociale, propriété de soi. Entretiens sur la construction de l'individu moderne. Paris : Fayard.

Castoriadis, C. (1975). L'institution de l'imaginaire. Paris : Seuil.

Dupuy, J-P. (2002). Pour un catastrophisme éclairé ; quand l'impossible est certain. Paris : Seuil. Durkheim, É. (1975a). La science sociale et l'action. Paris : PUF.

Durkheim, É. (1975b). Lettre à Célestin Bouglé (1898). Textes, Tome 2. Paris : Éditions de Minuit.

Durkheim, É. (1992). L'éducation morale. 3e édition. Paris : PUF.

Durkheim, É. (1998). De la division du travail social. 5e édition. Paris : PUF. Durkheim, É. (1999). Le suicide. 10e édition. Paris : PUF.

Ehrenberg, A. (2000). La fatigue d'être soi, dépression et société. Paris : Odile Jacob.

Ferry, L. (1992). Le nouvel ordre écologique. Paris : Grasset et Fasquelle.

Girard, R. (1982). Le bouc émissaire. Paris : Grasset. 
Girard, R. (2004). Les origines de la culture. Paris : Desclées de Brower.

Goffman, E. (1963, traduction française 1975). Stigmate ; les usages sociaux du handicap. Paris : Éditions de Minuit.

Heidegger, M. (1953, traduction française 1980). La question de la technique. Paris : Gallimard.

Hobbes, T. (1971). Léviathan. Paris : Sirey.

Jacques, F. (1982). Différence et subjectivité. Paris : Aubier. Jacques, F. (1985). L'espace logique de l'interlocution. Paris : PUF.

Jonas, H. (1985, traduction française 1990). Le Principe responsabilité. Paris : Cerf.

Kant, E. (1785). Fondements de la Métaphysique des mœurs - 2ème section, Victor Delbos, Alexis Philonenko. Paris : Vrin.

Legardez, A (2008). Co-construire des savoirs critiques sur l'environnement. Colloque « Éthique et éducation à l'environnement », 7 et 8 avril 2008, La Rochelle.

Legardez, A. et Simonneaux, J. (2007). Compétences et enseignement des questions socialement vives. Colloque « Compétences et socialisation », 7 et 8 septembre 2007, Montpellier.

Le Goff, J.-P. (2002). La démocratie post-totalitaire. Paris : La découverte. Lévinas, E. (1991). Entre nous. Écrits sur le penser à l'autre. Paris : Grasset.

Martinez, M.-L. (1997). Violence et réduction de la violence en éducation. Lille : Septentrion.

Martinez, M.-L. (2003). Sortir du pacte culturel violent, permettre l'émergence de la personne dans l'éducation. In Martinez, M.-L. (dir.), L'émergence de la personne, éduquer, accompagner (p. 19-43). Paris : L'Harmattan.

Martinez, M.-L. (dir.). (2003). L'émergence de la personne, éduquer, accompagner. Paris : L'Harmattan.

Martinez, M.-L. (dir.). (à paraître). Identités, éducation et formation à l'école. Paris : L'Harmattan.

Ricœur, P. (1990). Soi-même comme un autre. Paris : Seuil.

Sauvé, L. (2006-2007). L'éducation relative à l'environnement et la globalisation : Enjeux curriculaires et pédagogiques. Éducation relative à l'environnement : Regards - Recherches - Réflexions, 6, 13-29.

Serres, M. (1990). Le contrat naturel. Paris : François Bourin.

Weber, M. (1905). L'éthique protestante et l'esprit du capitalisme. Paris : Gallimard.

\section{NOTES}

1. Nous laisserons le débat sur les contenus et les approches didactiques et pédagogiques déjà traités par d'autres colloques de l'Institut de formation et de recherche en éducation à l'environnement et publiés dans de précédents volumes de la Revue Éducation relative à l'environnement: Regards, Recherches, Réflexions.

2. Sans doute, peut-on attribuer le désarroi des enseignants du premier comme du second degré, aujourd'hui en France, à cet effacement des finalités de l'éducation. À la place, on trouve une série d'objectifs quantifiés, peu lisibles, sans cesse revus et modifiés par des réformes successives dont la préoccupation semble exclusivement celle de réduire les coûts financiers.

3. Le dualisme est un système métaphysique selon lequel l'Être est formé de deux substances radicalement distinctes ; le monisme au contraire considère l'Être comme une seule substance. 
4. «La félicité de cette vie ne consiste pas dans le repos d'un esprit satisfait. Car n'existent en réalité ni ce finis ultimus, ni ce sommum bonum dont il est question dans les ouvrages des anciens moralistes [...]. La félicité est une continuelle marche en avant du désir, d'un objet à l'autre, la saisie du premier n'étant encore que la route qui mène au second [...]. Ainsi je mets au premier rang, à titre d'inclination générale de toute l'humanité, un désir perpétuel et sans trêve d'acquérir puissance après puissance, désir qui ne cesse qu'à la mort. » (Thomas Hobbes, 1971, chapitre XI)

5. «On se rappelle ce passage où Pascal essaye de faire sentir à l'Homme toutes les contradictions qui sont en lui. «S'il se vante, je l'abaisse, s'il s'abaisse je le vante, et je le contredis toujours, jusqu'à ce qu'il comprenne qu'il est un monstre incompréhensible ». En un sens, la morale fait de même. L'idéal qu'elle nous trace est un singulier mélange de dépendance et de grandeur, de soumission et d'autonomie. » (Durkheim, 1992, p. 104)

6. Pour illustrer la critique de l'individualisme actuel dans ses impasses, voir Ehrenberg (2000); Castel et Haroche (2001); Le Goff (2002).

7. Le "grand» individu, en lien avec l'autre et avec le monde, reste la personne. Produit de l'éducation et de la socialisation, la personne est la forme du sacré moderne (Durkheim, 1975a, p. 259-278).

8. «Il faudrait montrer que l'individualisme, quoiqu'on fasse, est notre seule fin collective; que loin de nous disperser, il est le seul centre possible de ralliement ; qu'il était déjà tout le continu positif, tout l'acquêt réel et durable du christianisme. Que l'individualisme bien entendu, n'est pas l'égoïsme, mais la pitié et la sympathie de l'Homme pour l'Homme et que je mets au défi qu'on nous propose une autre fin que celle-là. » (Durkheim, 1975b, p. 423)

9. «Cette personne humaine, dont la définition est comme la pierre de touche d'après laquelle le bien se doit distinguer du mal, est considérée comme sacrée, au sens rituel du mot pour ainsi dire. » (Durkheim, 1975a, p. 264)

10. «Dès que le pronom «je » apparaît dans un énoncé où il évoque -explicitement ou non- le pronom «tu " pour s'opposer ensemble à «il» une expérience humaine s'instaure à neuf et dévoile l'instrument linguistique qui la fonde. » (Benveniste, 1966, p. 228)

11. La praxis véritable est la pratique qui permet une construction identitaire du sujet soucieuse de l'autonomisation de l'autre (Castoriadis, 1975).

\section{RÉSUMÉS}

Partant de la nécessité d'un questionnement éthique, axiologique et praxéologique sur l'éducation à l'environnement et ses fondements, ainsi que de l'élaboration d'un modèle cohérent, l'objectif est d'y contribuer en articulant deux approches complémentaires : une approche anthropologique théorique critique et une approche anthropologique empirique. Dans un premier temps, l'article propose à cet effet l'examen des finalités, des valeurs et des pratiques, dans la perspective d'un modèle théorique global. Y est formulée l'hypothèse selon laquelle les pratiques éducatives associatives ou institutionnelles permettent une construction identitaire subjective et sociale dont les caractéristiques observables correspondent à celles des notions d'écocitoyenneté et d'écoresponsabilité. La seconde partie, à partir d'une étude empirique et descriptive, présente les effets de certaines pratiques du CPIE (Centre permanent d'initiatives pour l'environnement) des îles de Lérins et Pays d'Azur. Une analyse discursive des données langagières recueillies (entretiens d'enfants avec les animateurs) identifie les caractéristiques 
lexicales, thématiques, conversationnelles, relationnelles, psychoaffectives et psychosociales des identités subjectives et sociales, co-construites dans les interactions langagières. Des remarques sur les limites de la démarche permettent d'envisager quelques pistes pour l'améliorer.

Considering the need for an ethical, moral and praxeological analysis of environmental education and its foundations, as well as the need for the development of a coherent model, two complementary approaches are articulated : a theoretical critical anthropological approach and an anthropological empirical approach. First of all, the paper offers an analysis of its goals, values and practices, within the perspective of a comprehensive theoretical model. The assumption is made that the educational practices of institutions or organizations, allow for the construction of a subjective and social identity whose observable characteristics are in line with those of the concepts of eco-citizenship and eco-responsibility. The second part of the paper, based on an empirical and descriptive study, highlights the effects of certain practices carried out by the Lerin Islands CPIE (Permanent Center for Environmental Initiatives). A discursive analysis of the language data collected (children interviews with facilitators) identifies lexical, thematic, conversational, relational, psycho-emotional and psycho-social characteristics of subjective and social identities, co-constructed in language interactions. Remarks on the limitations of the method offer some ideas for improvement.

\section{AUTEURS}

\section{MARIE-LOUISE MARTINEZ}

Maître de conférences en sciences de l'éducation à l'Université de Nice Sophia Antipolis, à l'IUFM. En tant qu'enseignante, elle contribue à la formation des maîtres du premier et du second degré et des travailleurs sociaux. En tant que chercheur, elle est rattachée à l'UMR ADEF (apprentissage, didactique, enseignement et formation). Ses travaux de recherche portent sur l'analyse de la violence et de la sortie de la violence, sur le plan social et sociétal, ainsi que sur la construction des identités personnelles, sociales et professionnelles en éducation et formation. Depuis 2004, elle participe à des recherches fondamentales et appliquées (soutenues par l'ADEME ou par l'ANR) sur l'éducation à l'environnement et sur l'écocitoyenneté et l'écoresponsabilité.

\section{FRÉDÉRIC POYDENOT}

Directeur du Centre Permanent d'Initiatives pour l'Environnement des Îles de Lérins et Pays d'Azur, Docteur en Océanologie et médiateur scientifique, il développe l'éducation à l'environnement sur le milieu marin et littoral depuis 1996. Il est impliqué dans l'animation des réseaux des CPIE, GRAINE PACA, UFCV et RAMOGE. Il contribue à la création d'outils pédagogiques de sensibilisation à la protection du milieu marin en partenariat avec le Réseau mer PACA. Il participe à la formation des acteurs de l'environnement. Le Centre Permanent d'Initiatives pour l'Environnement réalise des actions d'éducation à l'environnement pour la jeunesse et tous les publics sur l'île Sainte Marguerite à Cannes, au jardin botanique de la Villa Thuret à Antibes et dans les établissements scolaires en partenariat avec les organismes liés à l'environnement. 